\title{
A method for bringing user experience upstream to design
}

\section{This paper intends to correlate human emotional response to product physical characteristics}

\author{
Monica Bordegoni, Umberto Cugini, Francesco Ferrise* and Serena Graziosi \\ Politecnico di Milano, Department of Mechanical Engineering, Milan, Italy \\ (Received 7 April 2014; accepted 11 June 2014)
}

\begin{abstract}
In the product development process one of the crucial phases is the evaluation of the design of the product that must satisfy the marketing targets based on the users' needs analysis. It is commonly acknowledged that a product is successful if people like and buy it. In the phase of ideation of a new product, it is paramount to test functionality and performances as well as the users' appreciation and feeling towards the new product. More specifically, in the case of consumer products characterised by a plurality of offers, interaction and experience should be addressed in addition to function and aesthetics in the user studies. Recent research has focused on the study of the user's emotional reaction when interacting and experiencing products, which is correlated with the global appreciation of the product and of its attributes. This paper presents an emotional engineering methodology using interactive virtual prototyping for evaluating the user experience and the emotional response with newly designed products early in the development process. The methodology suggests a way to optimise those aspects at the product concept phase.
\end{abstract}

Keywords: virtual prototyping; product experience; emotional engineering

\section{Introduction}

The history of a product that we buy at shops goes through many phases. The whole history is named the product develop-ment process (PDP). The marketers are those who trigger the process: they perform research on consumer preferences and expectations aiming to find out new needs and motivations for a new product (Roozenburg and Eekels 1995). Subsequently, a rough idea of the open space for the new product potentially available on the market is illustrated to the product designers, who start creating concepts representing the new product, including the definition of its shape, architecture, materials, functions, and also address some issues like ergonomics and usability. The typical subsequent step consists of the product designers and the marketers analysing and evaluating the concepts together, and agreeing on the selection of one concept, which will be the future product, which is transferred to the

*Corresponding author. Email: francesco.ferrise@polimi.it engineering designers, who check the feasibility and costs, and transform the concept into detailed technical specifications.

The concept proposed by the product designers may be subject to many modifications, mainly because engineers have to cope with various technical, economical and production constraints that may contrast the designers' choices. But an additional key issue to consider is the fact that marketers and designers express their idea about a product and its features by means of abstract, vague, and non-detailed descriptions, or even through similarities with the features of other products. Therefore, the probability that the product eventually detailed by the engineering designers is the one that marketers and product designers had in their mind is very unlikely, at least at the first design round.

For the above mentioned reasons it is paramount to set up a validation practice, which can be used to review and modify the design, if necessary. The product development process includes many loops, back and forth between the various 
phases, which are required by the necessity to change the design due to engineering constraints, or because of nonsatisfactory validation results. The validation practice can address product technical characteristics and performance, as well as issues more related to users. In particular, when dealing with consumer products, some considerations about consumers, i.e. the future users of the product, and their relation with products should be made as well. In fact, users interact with products, during their daily use but often also during the purchase. And both these aspects are important to be considered from a marketing analysis point of view. In this respect, the interest of both industry and research has recently been focusing on the study of the user's emotional reaction when interacting and experiencing products, which is correlated with the appreciation of the product and of its attributes.

An issue we are interested to address in our study concerns how we perceive products, and what are our elicited emotions and emotional behaviours during the interaction with products. In so far as we are able to understand the human emotional response with respect to some product attributes, we can plan to tune those attributes until the emotional response is the desired (positive) one. In fact, designing products with the intention to evoke or to prevent elicitation of certain emotions can be facilitated by an understanding of emotional processes (Demir et al. 2009).

This paper investigates our experience with products, how we perceive a product through our sense and multi-sense channels, and which kind of emotions we feel during the interaction with a product. These studies are at the basis of an emotional engineering method that we have developed for defining product attributes according to the users' emotional responses. The method is based on interactive virtual prototyping that allows us to change a product's attributes and consequently its behaviour by simply modifying the parameters driving the functional model of the virtual product.

\section{User experience}

The major question at the basis of the decision to develop a new product is 'Is there a need for such a product on the market?'. In general, for a product to sell well you have to make sure that it is good. This is also related to whether people like that product, and associate a high value to it, so as to induce them to prefer and buy it. Therefore, predicting the value perceived by users is crucial in the process of development of new products. Yet value is a very intangible and personal dimension only partially correlated to the physical objective characteristics of products.

A product has several attributes and properties, and those must not only meet the users' needs, but also satisfy their tastes and preferences. A feature characterising consumer products we are interested to study is interactivity. In fact, people typically use these kinds of product through an active, dynamic interaction. The interaction is related to the action of using the

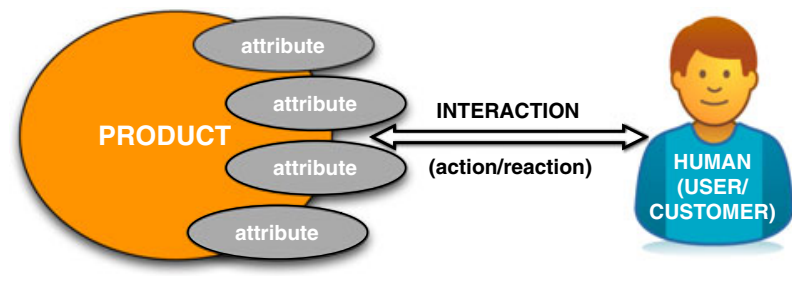

Figure 1. Interaction correlating human and product.

product, which provokes reactions in the humans. Reactions, both conscious and unconscious, elicit new actions, and so on and so forth. Humans can be considered as the customers at the moment of deciding whether to buy a new product, among a set of available alternatives, or users who use the product after the purchase (Figure 1).

Several frameworks describing the perception of artefacts have been developed. Norman has developed a framework based on three levels of processing, including visceral, behavioural and reflective levels, which all humans have in common (Norman 2004). In the context of the interaction of users with products, recent studies have addressed User Experience (UX), which aims to measure the effectiveness, usability and satisfaction that products provide to users (Hassenzahl 2013). UX involves studying a person's objective performance, and a person's subjective emotions when using a product. The first mainly addresses usability issues when users interact with a product. It measures how easy using a product is, errors made, comfort etc. The latter is more focused on studying the feeling and emotional responses when interacting with a product. In this last case, user experience investigates the experiential and affective aspects of human-product interaction influencing the ex-post global evaluation.

The study of user experience can be performed considering a typical use of a product, as it would be used on a daily basis, but can also consider the first impression that we have when experiencing a new product in a shop, for the first time. In fact, it is interesting to study the interaction of a potential customer with a product at the time of purchase. Beyond the fact that people like a certain product and give great value to this, it is also important that they eventually decide to buy exactly that product, and not others maybe proposed by competitors. So the key point is understanding how people decide to buy a product, i.e. how customers are influenced and driven to their buying decisions. And this issue is very much related to understanding how we make decisions, a topic that is extensively studied in the marketing domain (Kahneman and Tversky 2000).

The purchase experience is driven basically by one of two factors: the product is a need, or it is a product of desire. For example, we need a new tube of toothpaste because the current one is finished. Differently, we may decide to buy a new pair of shoes, even if we own many. We simply want a new pair of shoes; this is a product of desire. The first seems a rational decision, the second an emotional one. 
Nevertheless, let's consider, as an example, a refrigerator. We need to buy a new one because ours is old and not working properly. So, we go to the retail shop with the intention to buy one, because we really need it. We may want one with low energy consumption, we have some space requirements, we want one we trust to last for some years etc. At the shop there are many refrigerators satisfying these requirements, which we consider of major importance; all these refrigerators are very similar - if not identical - in terms of functionality, but have different prices. So how do we decide which one to buy? The decision cannot be taken considering the functionality alone as all products are so similar. Actually, the products may have a very different symbolic or relational value, which contributes in differentiating their perceived value (Beckert and Aspers 2011).

The perceived value of products has been driven by the fundamental assumption of a complex reaction based essentially on features, functions, and cost. Customers buy products for their features (for example, a mobile phone with a touch screen) or their specific functionalities (for example, a mobile phone playing video and music). Similarly, potential customers make purchase decisions considering a product's price. That is the best perceived value in terms of benefits and cost.

Today these perceptions may not completely reflect reality. In fact, in a market that is offering plenty of very similar products, people tend to buy under the influence of the perceived intangible benefits that products deliver, directly and indirectly, that is on the basis of symbolic and relational value. Features and functions, which are the main focus of product design specifications, become important for delivering the benefits that are more desired by customers. For all products on the market that meet objective performance criteria, which are typically validated by analytical or physical lab tests, a product is successful only if the customers recognise that the product actually delivers some intangible benefits. And it is also worth noticing that the perception of the customer about the product is central in the purchasing experience.

As reported in many marketing studies, most of the time the purchasing decision is not logical or rational, but emotional. In fact, it seems that emotions greatly influence and, in many cases, even determine our decisions (Damasio 1994). Consumers form their value perception of one or more products or brands; and these perceptions are made up of objective and subjective attributes. In the example of the refrigerator, an objective attribute can be energy efficiency ranking $\mathrm{A}+++$ (according to the European Union energy label); subjective attributes may be brand prestige and reputation, or may be the feeling of robustness perceived when opening and closing the doors.

In summary, the main issues that people consider before placing a value on a product are the following:

- Attributes: They include objective and measurable characteristics like size and colour. For example, one consumer may prefer a white mobile phone cover to a black one.

- Functional characteristics: These can be related to the benefits that the consumer expects to gain from the product.
They concern all the advantages a product offers as compared to similar product offerings. For example, a product is easy to use, its design meets the expectations of the customer, it is easily available and it has a long lifetime. Some of them are objective and therefore can be measured and compared.

- Emotional effects: This refers to the feelings evoked in customers while and after buying a product. The quality and reputation of the brand as well as the characteristics of the product play an important role in stirring feelings in customers (Rao and Monroe 1989). The feelings can be contentment, anger, excitement etc. For example a lady decides to buy a dress according to how it fits on her body, and also for the tactile feedback provided by the fabric, and the style evoking or explicitly claiming the brand. Those are typically subjective, temporary and emotional effects.

It is important that all these issues are considered at the design level. While attributes and functional characteristics have been studied for years, using well-established methods, such as realistic rendering, functional simulations, usability studies etc., the emotional aspects have been addressed only recently, and appropriate methods are still under investigation.

The study of emotional effects is closely related to the user interaction with products, and with the experience that users have with products. Nowadays, the so-called User Experience Design (UXD), which is part of the design of a new product, is gaining ground (Hassenzahl and Tractinsky 2006). User experience design includes the definition of the interaction components, i.e. their shapes, material and layout, and the definition of their behaviour to users' actions. User experience design refers to the application of user-centred design practices to generate predictive and desirable designs based on the consideration of users' experience with a product. These practices include the definition of the user interface, graphics, physical and manual interaction etc., performed in close collaboration with target users.

It is paramount that the design team deals with the design of the experience of a person when using and interacting with a product, so as to optimise his/her enjoyment, satisfaction and positive emotional response. Actually, the optimisation is related to the definition of metrics and methods of measurement. Therefore, an issue we have to study is the objective detection of the emotional response of people when experiencing products for the first time, or even during their prolonged use. In order to study the emotional response, we first need to investigate how people experience products, which takes place through their senses, and the emotional experiences they undergo.

\section{Sensing products and emotions}

This section addresses issues related to how we sense products during interaction, and the emotions elicited in us. The first 


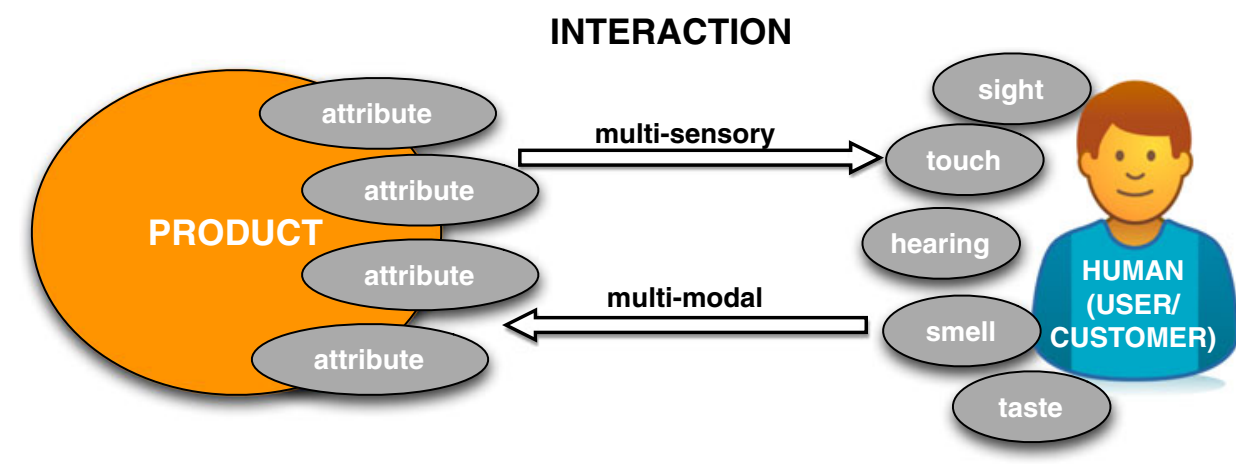

Figure 2. User interaction with products occurs through human multiple sensory channels.

consideration concerns the way we perceive things: it occurs through our multiple sensory channels (Figure 2). The sense of sight has been thoroughly studied over the years (Marr 1982). We hereafter focus on the sense of touch, sense of hearing and sense of smell, which have been recently investigated with the aim of developing technology for their simulation.

\subsection{Sensing products}

The sense of touch is the first to develop in humans, well before vision (Field 1995). In a general sense, touch plays an important role at both the emotional and perceptual level, even when the user is not aware of its use (Gallace and Spence 2013). As a confirmation of this, some researchers have addressed the so-called touch hunger. This concept, introduced by Tiffany Field, refers to the fact that many people report the lack and the negation of tactile stimulation in society today, thus also causing psychological problems (Field 2003, Gallace and Spence 2013).

The sense of touch is also important when interacting with objects. In particular, through our experience of touch, important features of an object are transmitted to the users, such as the surface texture properties, the product shape, dimension, overall quality etc. Besides, touch experience has a very evocative effect.

In recent years, industry has extended the concept and the communication of their products, by exploiting other sensory modalities such as sound and smell with the aim of effectively anchoring a company's message with an individual on the sensory level. In fact, it is recognised that 'the bond of an individual to a company (brand, product or service) is driven up to $90 \%$ by emotional-subjective values and by a corresponding small portion of rational-measurable requirements' (Stompff 2003). Consequently, the interest of the research has become wider, including multi-sensory interaction with products in the studies.

Several research studies have demonstrated that sounds can be used to elicit users' emotions. Lu and Petiot have compiled an emotional space of sound, classifying sounds as relaxing, peaceful, disturbing, and aggressive ( $\mathrm{Lu}$ and Petiot 2012). Some examples using sound for conveying a product distinct attribute have been developed, particularly in the automotive domain. For example, sounds have been developed for car door closing (Bezat et al. 2007), or for electric vehicles (Petiot et al. 2013).

Recently, a number of companies have started to use smell to sell their products. The subtle smell of coffee may make us more likely to buy a cup, or the smell of clean cloths may lead us to buy a specific washing machine. Several retail companies already use scent in order to attract their customers. In fact, it seems that specific scents keep people in stores longer, and also may lead them to spend more money. A scent logo can be effectively added to establishments, shops, or even to any kind of products that do not naturally have a scent, such as for instance clothes. A certain smell can bring to mind an experience, thanks to the strong links that exist between smell and memory, which works as a powerful reminder. In addition, it has been discovered that the sense of smell is closely connected to the parts of the brain responsible for processing emotions (Schacter et al. 2011). Furthermore, it is generally acknowledged that emotional reactions make our experiences deeper and more engaging.

In our everyday lives, perception occurring through multiple sensory channels allows us to undergo emotional experiences. The next section reports studies about emotions.

\subsection{Emotions}

Many definitions of emotions have been given. According to the Webster Dictionary emotion is defined as: 'A conscious mental reaction (as anger or fear) subjectively experienced as strong feeling usually directed toward a specific object and typically accompanied by physiological and behavioural changes in the body.'

In 1984 Scherer defined an emotion as: 'Relatively brief episode of synchronised response of most organismic subsystems in response to the evaluation of an external or internal event as being of major significance' (Scherer 1984). 
Recently, many research works have addressed emotions and the relations of emotions with the perception of the world, have classified emotions and have also defined methods for measuring emotions. In the context of our research, we are interested in understanding how we can detect and measure the type and intensity of the users' emotions, in order to be able to objectively compare the effects of design product attributes that elicit specific emotions.

In recent years, several methods and technologies have been developed to measure human emotion and sensibility, including:

- psychological methods, mainly based on self-reporting;

- behavioural methods, based on the analysis of facial expression, gesture, and posture;

- physiological methods, detecting emotions by processing human body measured parameters such as heart rate, skin conductance etc.

Actually, on the side of the emotion detection, in cognitive and in affective sciences, there is not yet an established and fully reliable method of measuring the experience of specific emotions (Mauss and Robinson 2009), despite recent increasing attention being devoted to issues related to emotions. Moreover, the specific theoretical model that should be applied to describe emotions is still under discussion. However, there is an increasing agreement that emotions can be the result of an appraisal process that leads all the other emotional components (Scherer 2001).

Briefly, appraisal theory describes an appraisal process as a continuous evaluation of the events surrounding the subject. This evaluation determines the relevance of an event for a person, and finally this relevance can influence the human's emotional evaluation of a given emotional stimulus related to the estimated event. Research agrees that the subject's evaluation of an emotional stimulus can be reflected by a series of components, including physiological responses, behaviour (expression and action tendencies) and subjective feeling (Scherer 1984). Emotions should be investigated looking at their multiple components at the same time, and this implies the use of different measures (Zurloni et al. 2011).

Indeed, emotional components have been investigated through many different techniques.

First, self-report measures, i.e. the Self-Assessment Manikin (Bradley and Lang 1994), have been used for many years and still represent one of the best ways to access the conscious experience of an emotion. However, there are limitations, because the obtained measures considerably depend on the kind of questionnaires used to assess the human emotional states (online vs. recall of past experiences) and on the individual characteristics of each person (Barrett et al. 1998).

Measures of the activity of the central nervous system (by means of Electroencephalography [EEG], fMri) have been used for assessing the existence and the location in the brain of the appraisal process activity and the subjective feeling (Grandjean and Scherer 2008). Then, measures of expressive behaviour, that include the description of facial expressions, bodily movements (Castellano et al. 2008), and vocal nonverbal parameters, have been developed. Research for each of these modalities of expression of the emotions has been conducted so far mainly in a separate way, so that no multimodal study of emotional expression exists. Facial expression is the most studied modality and probably the one for which the more reliable results are available (Ekman 2007): this is also due to the existence of a widely agreed upon coding system (Ekman and Friesen 1978). The analysis of vocal expression of emotions has advanced at a slower pace, but available results show that there is statistically significant correspondence between some acoustic features and emotions (Juslin and Scherer 2005). However, research on voice and appraisal is very limited (Johnstone et al. 2005).

Finally, theories of emotion acknowledge differential physiological response patterns as an important outcome of emotion (Ekman 1999, Scherer 2001). The majority of research has focused predominantly on physiological changes associated with negative emotions, such as fear, anger, sadness, and disgust (Ekman et al. 1983). In this context, a number of important response measures have been identified, such as heart rate variation, respiration measures, and skin conductance level, as well as activation of certain facial muscles measured using electromyography (Cacioppo et al. 2000). Less, but still present, research has instead addressed the physiological effects of positive emotions, such as joy, happiness, pleasure, or pride, where the first evidence of differential response patterns has been found (Bradley and Lang 2006).

In our research group, we have developed a framework recording four different types of physiological signals, usually associated with an emotional activation: skin temperature, respiratory activity, cardiovascular activity, and skin conductance (Gatti et al. 2013). Sensors are placed on a person's body (for example, respiration sensor is placed around the person's chest, Galvanic Skin Response sensor and biofeedback sensor are placed on the person's skin). The framework also acquires images of a person's face. Meanwhile recording the physiological features and the facial expressions, the framework also allows the experimenter to control several features of the experimental set-up, such as lighting, ambient smell, music, and stimuli displayed on the screen that can be either pictures or movie clips, and audio tracks. The program also gives the participants the possibility to interact with the stimuli displayed on the screen, modifying their features (for example, changing volume and luminosity intensity for the movie clips) and answering the question the experimenter might be interested in. Emotions previously classified are associated with specific patterns of data sensors.

In our study we are interested to understand the correlation between some product attributes and the elicited emotions (Figure 3). Capturing this correlation would allow us to figure 


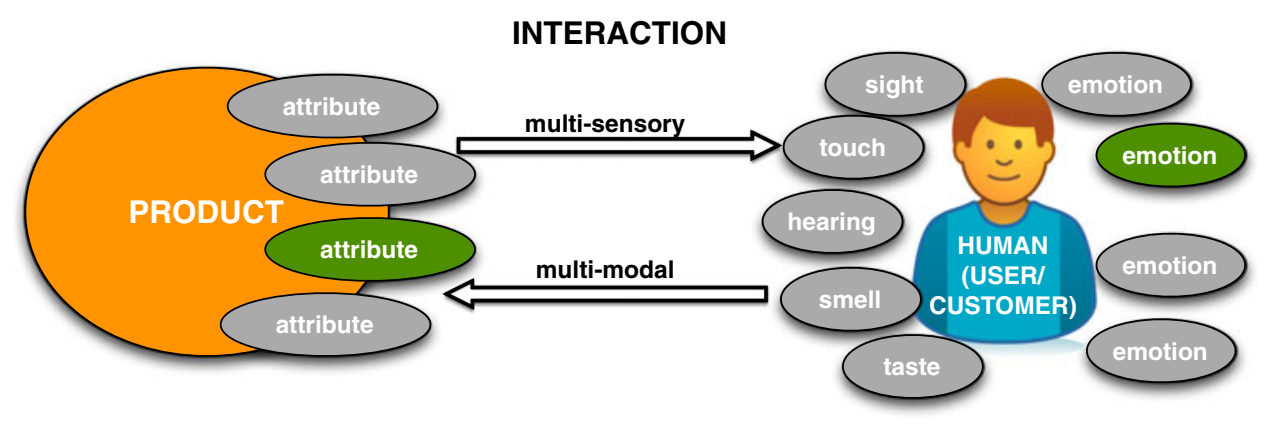

Figure 3. Correlation of product attributes and elicited emotions.

out how a product can be designed in order to evoke elicitations of certain emotions. These issues are discussed in the following section.

\section{Emotional engineering}

In order to design a product that elicits positive emotions, it is necessary to define and be able to predict the target users' emotional responses early in the design process. In other words, the aim is to understand which are the more relevant and influential attributes or characteristics, and how we can correlate these attributes and properties of a product with the elicitation of certain emotions.

Emotional engineering is a recent discipline that has introduced methods for predicting and likely being able to control users' emotional responses with respect to product attributes, in order to be able to design and engineer them (Fukuda 2011, 2013).

One of the first pioneers of emotional engineering studies has been Mitsuo Nagamachi, who defined emotional engineering as 'the technology to design goods which appeal to emotion and sensibility by translating human sensibility and images into physical design factors' (Nagamachi 1997). From then, the research on emotional engineering has focused on several topics, has been applied to various domains, and has addressed several disciplines.

Initially, the research focused on studying new methods to design products that elicit positive emotions in future users, addressing the study of the visual perception of the products. For example, the Kansei methodology mainly correlates emotions with the visual perception of shapes (Nagamachi 1995). Traditional Kansei engineering approaches use items and categories defined in pictographic terms to provide qualitative descriptions of the global product shape; they may support the conceptual design of a product by facilitating the creation of product variations. Surveys or experiments are conducted to evaluate the consumer's image of the product. With the identification of the design elements of the product, the relationship between Kansei (image) words and the design elements can be established (Osgood et al. 1957).
Due to the increasing complexity of products, and the fact that most of the products are actually used, besides being watched and admired, the definition of emotional engineering gains a wider perspective. Therefore, with respect to Nagamachi's definition, we define emotional engineering as a methodology enabled by most advanced IT technology, to design products that appeal to emotions by translating human sensibility and multi-sensory effects, including images, sounds, haptic/tactile effects and smells, into physical design factors. In other words, emotional engineering applies scientific and engineering methods to identify and analyse the relevant human emotional responses, by providing quantitative parameters that feed the design phase.

The following sections describe the emotional engineering methodology we have developed, and its integration in the product development process. Besides, the sections present interactive virtual prototyping, which is a method for early user experience evaluation based on state-of-the-art virtual reality technologies.

\subsection{Emotional engineering methodology}

Recently, several studies have focused on the emotions elicited in humans during interaction with a product (Demir et al. 2009). In particular, emotional engineering is a discipline that studies quantitative parameters of the future product (attributes of the product-to-be) that generate physical and physiological effects during the interaction, which are perceived as emotionally relevant. The design process aims at optimising these parameters, in order to elicit intended (positive) emotions.

Emotional engineering (EE) is multi-disciplinary and multidomain in its nature. It is related to two disciplines: product design and cognitive sciences. The first discipline is focused on the methods and process for the ideation and creation of effective and possibly successful new products; the latter deals with human aspects during the interaction with products, including the study of perceptions, behaviours, feelings, reactions etc. Therefore, by synthesising, EE deals with the interaction of humans with products.

Figure 4 shows a representation of the three disciplines design, cognitive sciences and emotional engineering - and 


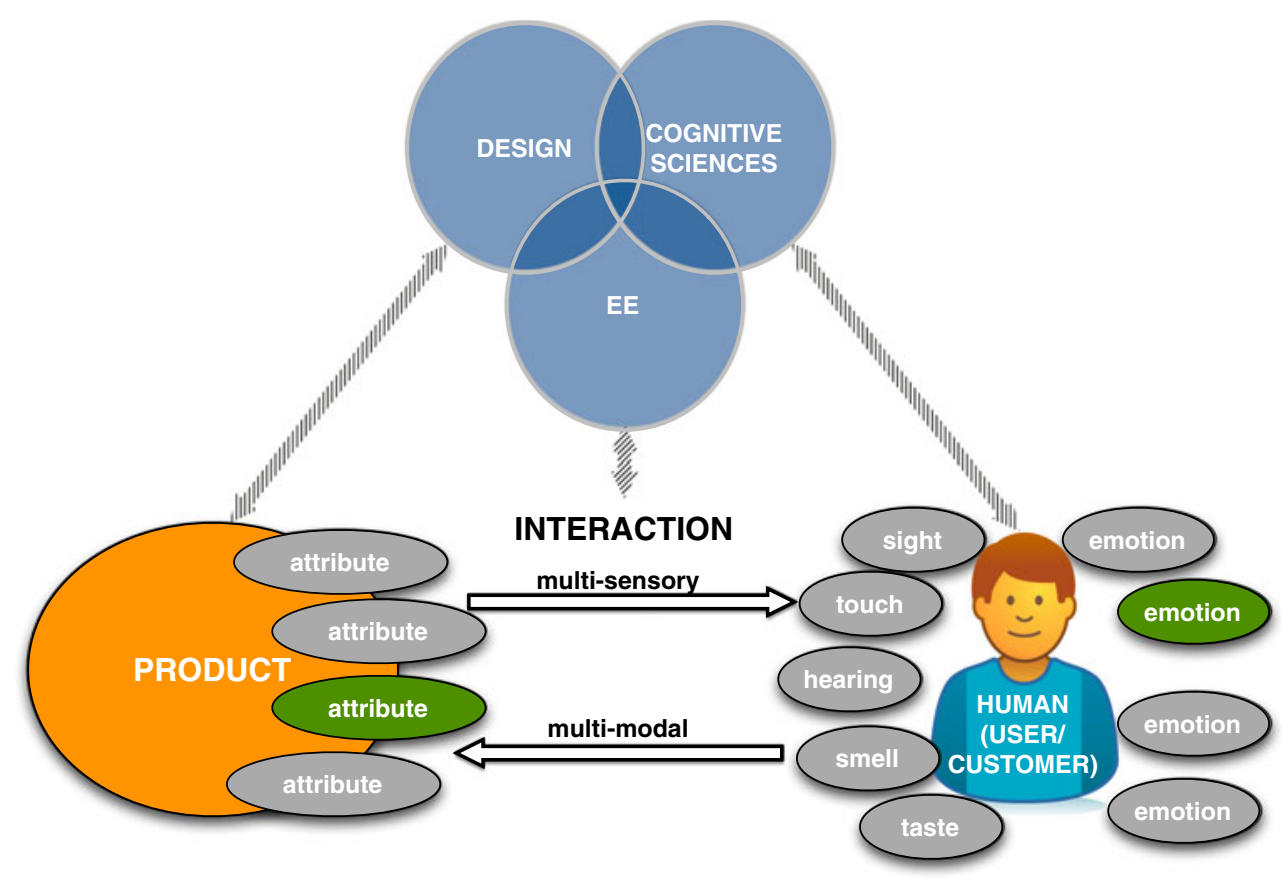

Figure 4. EE, design and cognitive sciences: three intersecting disciplines.

their relationships with the product and human, and the interaction of the two of them.

The three disciplines have areas of intersection, addressed by specific research domains, which are the following (Figure 5A):

- User-centred design methods. The intersection of design and cognitive sciences identifies the area of user-centred design methods, which are those methods for the product

design that start from the users' needs and expectations. The research on design methods has developed, among others, the User-Centred (UC) Design theory (Hassenzahl and Tractinsky 2006), where the designer, in collaboration with users, evaluates the quality of the developed design solution and, possibly, modifies it until reaching the desired solution in terms of 'external' behaviour, which users 'like more'. In this process, together with the evaluation of the usability of the product, the evaluation of its aesthetic form takes place as well, both using visual and tactile exploration and perception.

- Evaluation/appraisal. The investigation and study of methods, and the comparison and appraisal of products can be placed at the intersecting point between EE and cognitive sciences. The research in this area has focused on the definition, experiment and validation of methods and tools to measure human emotion and sensibility, which are more objective and reliable than subjective qualitative selfassessment, and which can generate quantitative physical design specifications. Several technologies for the measurement of human emotion and sensibility have been developed, including technologies for physiological signals detection, facial expression detection etc., as described in Section 3.2 (Ekman 2007). In addition, it is necessary to understand how the symbolic and relational value is built, in relation to the context.

- Simulation. Product simulation is a well-established design method traditionally focusing on analytical or physical laboratory tests of the 'internal' behaviour of a system as a whole, or of the behaviour of single components and their interaction (Bordegoni 2011). It is used to check the robustness, reliability and efficiency of the detailed design of the product, and of its components. In our diagram, simulation is at the intersection point between EE and product design, and is intended to be focused on the 'external' behaviour of products, that is the one that is experienced by the users. The research is aimed to explore and test in advance the full space of possible product behaviours during interaction and product use in simulated environments, with the aim of capturing and measuring the human emotional responses, without the cost of performing experiments with real prototypes. This requires a method to globally model the product, without the necessity to develop a detailed design.

At the intersection of all disciplines are the specific Applications and Case Studies (Figure 5B). In fact, the research results are applied into the making of specific products or services, in a given context. Several applications have been developed including the various evaluation and simulation methods, by addressing many contexts such as 


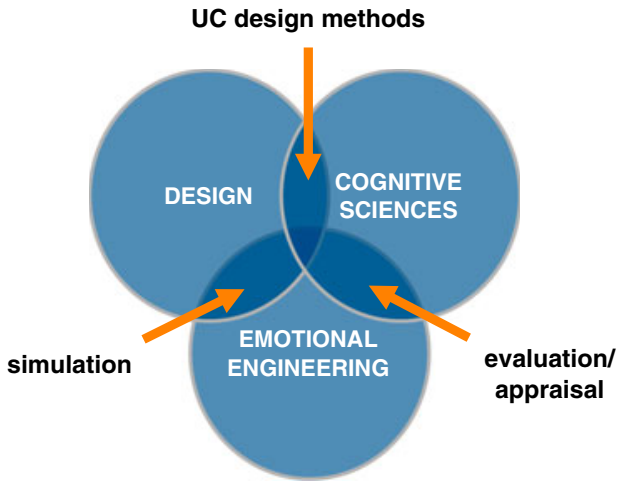

A. Research Areas

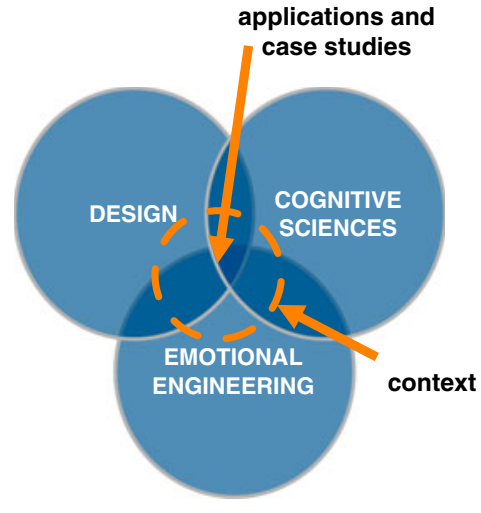

B. Applications and Context

Figure 5. A) Research areas in the domain of emotional engineering. B) Applications and context of the research.

automotive, consumer and electronic products etc. (Fukuda 2011, 2013).

In our research we are interested in defining methods to use in

a proposed New Product Development (NPD) process for simulating the product behaviour, evaluating the target users' response, and measuring the appraisal.

\subsection{Emotional Engineering in the NPD}

As stated in the previous section, emotional engineering studies quantitative parameters of the future product that generate physical and physiological effects during the interaction, which are perceived as emotionally relevant. Therefore, a major issue to face in the New Product Development (NPD) process (Blessing and Chakrabarti 2009) is how to define certain quantitative parameters of the future product, and check if the designed experience is satisfactory for the target users. However, this issue cannot be easily addressed, since it requires a properly functioning product that one can use for testing, before the real final product is available.

Simulating the product and its behaviour is one of the methods proposed for the evaluation of the product design, and of the user experience as well. Typically, simulations are based on the use of prototypes, and can be used for verifying and validating the behaviour of a designed product with people (potential customers and target users). It is possible to perform the simulation of a product, and use it for its evaluation only after the completion of the detailed design of the solutions that respond to the user's needs and requirements. This is a typical design process (Cuffaro and Zaksenberg 2013), which is represented schematically in Figure 6.

After conceiving the product (concept design), and detailing its design (engineering design), a physical prototype (PP) or a virtual prototype (VP) is built for the evaluation of the design.

The physical prototype consists of a physical artefact that is very similar to the final product. It can be experienced by users through their senses, it can also be physically touched, manipulated and operated. Users can formulate a judgment and give an evaluation about the product and its attributes. Users also feel emotional reactions, which contribute in determining the perceived value assigned to the product. The users' satisfaction and emotional response can be detected through questionnaires, or even through objective modalities, such as by using one of the evaluation methods described in Section 3.2. If the perceived value, which also corresponds to the level of acceptance and pleasantness of the product, is low it may be necessary to make some modifications to the design,

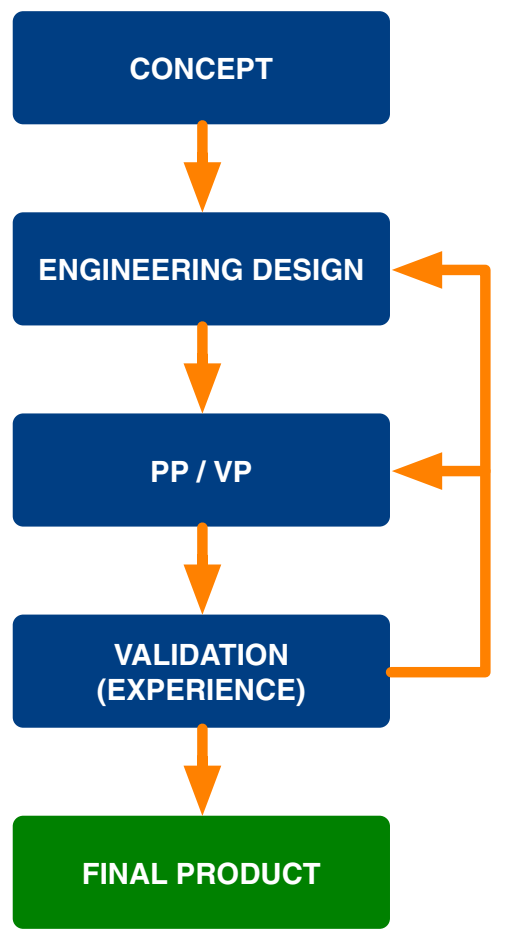

Figure 6. Typical product design process (PP, physical prototype; VP, virtual prototype). 


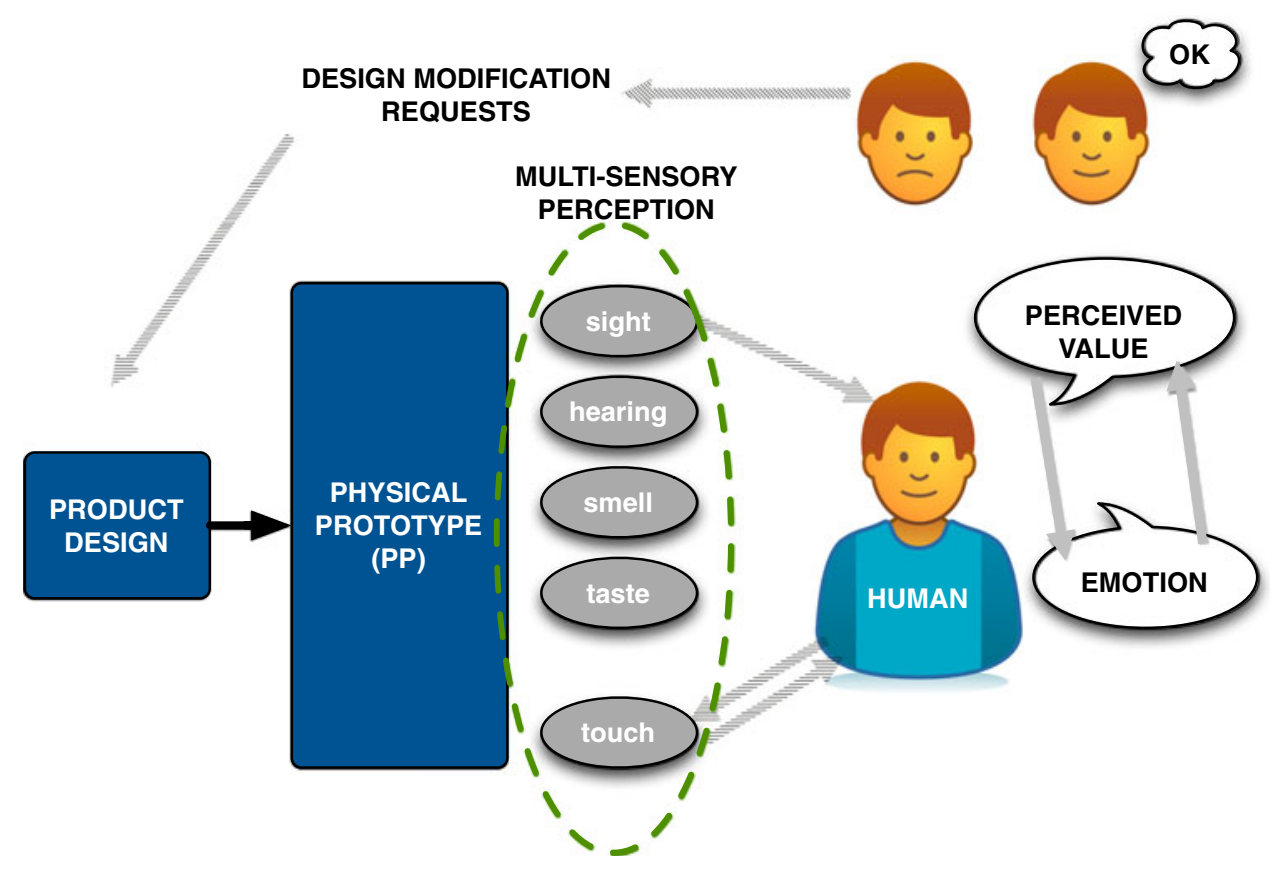

Figure 7. PP - Physical prototype for the evaluation of a product design.

and physically modify or remake the prototype, in order to increase the perceived value. In order to do that, it is necessary to be able to identify the sources of low acceptance, and correlate those with the design parameters that can improve the acceptance rate in technical and engineering terms (Figure 7).

As an alternative to the realisation of a physical prototype, which has high cost and may require long development time, today it is possible to develop a virtual prototype, which is made of three-dimensional (3D) digital models. The perception of the attributes of the product and the interaction with them are simulated through state-of-the-art virtual reality technologies (Bordegoni 2011). To be effective, the interaction with the simulation of the product should be multi-modal, in the sense that the users can use multiple modalities to interact with the virtual product, as well as multi-sensory, in the sense that the users can experience the product through their senses, as happens in the real world (Figure 8). Also in this case, the design, and the virtual prototype accordingly, must be modified if it does not meet the users' expectations. Indeed, the modifications performed on a virtual product are easier and faster to implement compared to the same ones made on a physical product. And this is one of the reasons why it is preferred, when possible, to evaluate and test the design of a new product by using virtual instead of physical prototypes.

\subsection{Interactive virtual prototyping}

Recently, the authors have proposed an emotional engineering methodology based on interactive virtual prototyping (iVP), which has been demonstrated to be effective for the evaluation of the user experience in the early design phase (Bordegoni et al. 2013).

Interactive virtual prototyping consists of the simulation of the behaviour and use of a product, which allows the evaluation of the user experience, and the compilation of a list of specifications to use for the product design (Bordegoni 2011).

Some attributes and interactive behaviours can be simulated and tested starting from the very preliminary ideas about how a product should be, and how it should behave. The methodology aims at giving the designers the possibility of evaluating in real-time the users' experience during the initial conceptual design of a new product. The focus of our study is on the interaction of the users with the newly conceived product, and on the effect that this interaction has on users, in terms of satisfaction and emotional responses. The aim is obtaining in advance, and predicting in simulated environments the human emotional reactions.

The methodology allows testing multiple interaction experiences and external behaviours, and identifying the ones that people like more. This methodology can be used to test proposed novel interactive solutions with groups of target users, when the product, not yet fully decided and designed, is at the concept level. Interactive virtual prototyping allows users to experience a product, or some of its sub-systems, through multiple sensory modalities. For example, it is possible to allow target users to test a new method for operating a coffee machine, or the physical response of a new refrigerator door.

In order to allow this kind of simulation, which requires a simple and fast modification of the product behaviour, the iVP 


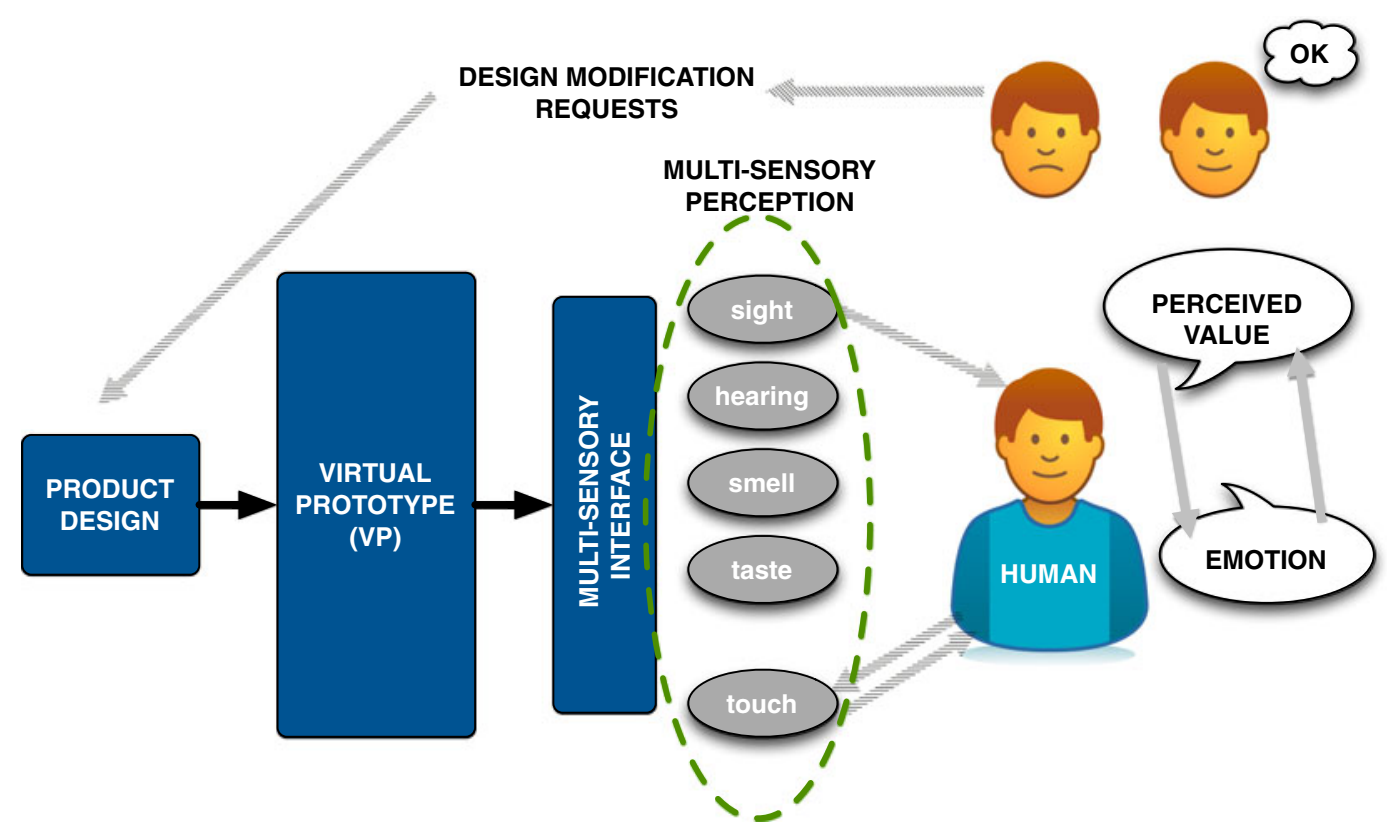

Figure 8. VP - Virtual prototype for the evaluation of a product design.

cannot be based on the simulation of a detailed design, which does not exist at this stage. Instead it is based on a simplified perceptual representation of the product, which is not a detailed model of the product, but is a model allowing us to simulate and generate those effects that one is interested to study and analyse. We define it as Perceptual Mock Up (PMU).

The PMU is based on the definition of several elements, one for each effect that we intend to simulate (i.e., vision, sound, and touch). A set of parameters is used to drive the behaviour of each element. During the tests of an interaction effect, when a user requires some changes, these can be actuated by modifying the values of the parameters driving the functions, until the desired effect is obtained. The PMU is a mathematical system, allowing us to switch among alternative solutions that are required for matching the behaviours and effects preferred by the users.

The benefit of using the iVP approach based on a perceptual mock up, with respect to virtual prototyping, is that in the first case changes are made at the perceptual level. This means that, once the iVP is consolidated, the PMU can be translated into quantified specifications of the desired product behaviour, in terms of physical performances, which can also be considered the precise target for the detailed engineering design.

Therefore, with reference to the typical product design process (Figure 6), soon after the concept is developed, it is possible to build a perceptual mock up, which is used to simulate the effects of the solution (Figure 9).

This new process is beneficial for capturing the effects causing the most positive emotional responses. Therefore, the iVP enables us to study and evaluate not the product functionality but the experience, and to get results about the emotional appraisal and the perceived value of a new product under development (Figure 10). The definition of the quantitative technical objectives of the detailed design phase will guarantee that, if the physical prototype behaves as specified, the product will be fully enjoyed by the potential customers, and therefore will most likely be successful.

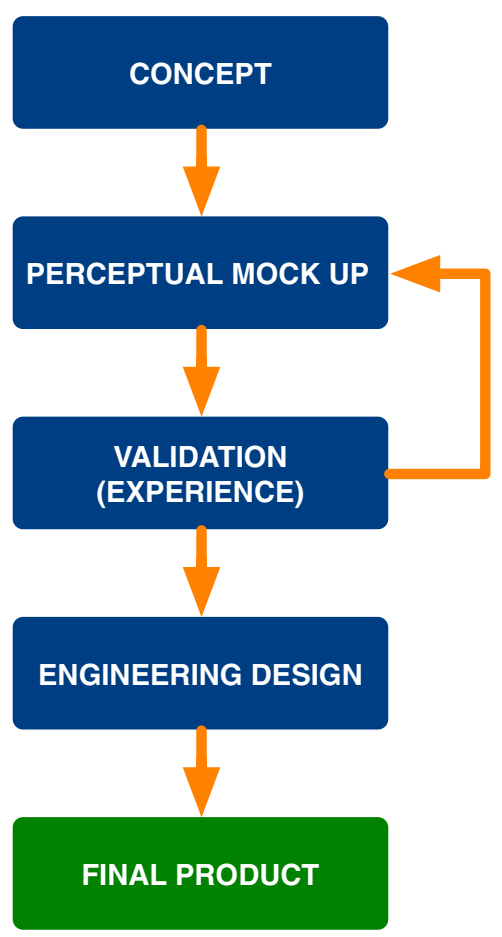

Figure 9. New product design process including the evaluation and testing based on the perceptual mock up. 


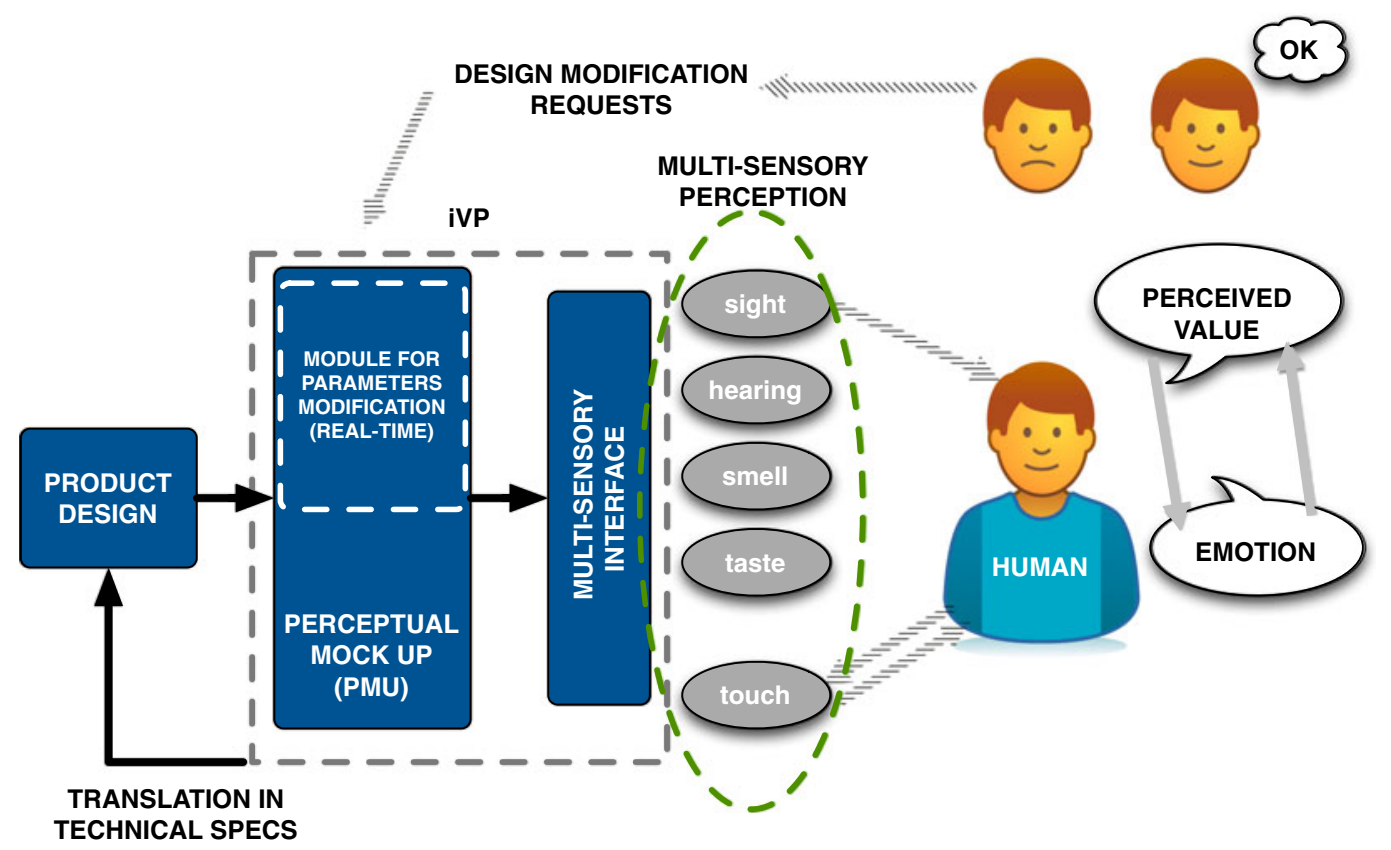

Figure 10. Interactive virtual prototyping based on PMU (perceptual mock up) for the evaluation of the user experience.

\section{Case study}

This section presents a case study demonstrating the use of the emotional engineering methodology previously described. The case study consists of building the interactive virtual prototype of the door of a refrigerator for testing the user experience, and their emotional responses (Ferrise et al. 2013). The motivation for selecting this use case is the following. The door of a refrigerator is the interface enabling the user to access the goods stored inside. Opening and closing a refrigerator door is a common gesture that one performs, usually, several times a day. Moreover this opening and closing activity is the main action a potential customer performs at the point of sale.

Domestic appliance manufactures know very well how much this first impact/interaction with the product is important at the point of sale for influencing the buying decision. Hence, improving the pleasantness of the opening/closing of a door or of a drawer is an important part of the overall product experience, and it is now becoming a strategic design requirement to satisfy. Consequently, in the process of designing a new refrigerator, the design team faces two issues:

- mapping the entire dynamic behaviour of the product interfaces (the door in this case) considering all the intermediate states they can undergo during the interaction, because each state influences the user's product perception;

- assessing the product reaction/perception (the one that is transferred to the user through the interface in relation to a variable input): different users can interact with the same product and its reaction should be able to elicit a positive perception on them.
The hypothesis made in this research work is that the proposed methodology can satisfy both these issues. A first experimental study is hereafter presented.

\subsection{Interactive virtual prototype of a refrigerator door}

A detailed physical description of the refrigerator door has been implemented using the LMS-AMESim suite (www. lmsintl.com) representing:

- the effect of the magnetic force of the door when it is closed;

- an hypothetic effect of a spring forcing the door to close after a certain angle;

- the elastic and damping effect of the gasket when opening/ closing the door;

- a click effect;

- the de-pressure-like effect generated by the air contained in the refrigerator and its temperature, the friction and inertial effects generated when opening/closing the door.

In parallel a perceptual mock up has been defined, which consists of simplified parametric equations in order to be interfaced with selected virtual reality technologies for simulating the multi-modal and multi-sensory interaction. Specifically, a 3D stereo wall display, a haptic device, and sound speakers have been used. A user interacts with the interactive virtual prototype of the refrigerator through these technologies as shown in Figure 11. More details about the development of the iVP of the refrigerator door are reported in Graziosi et al. 


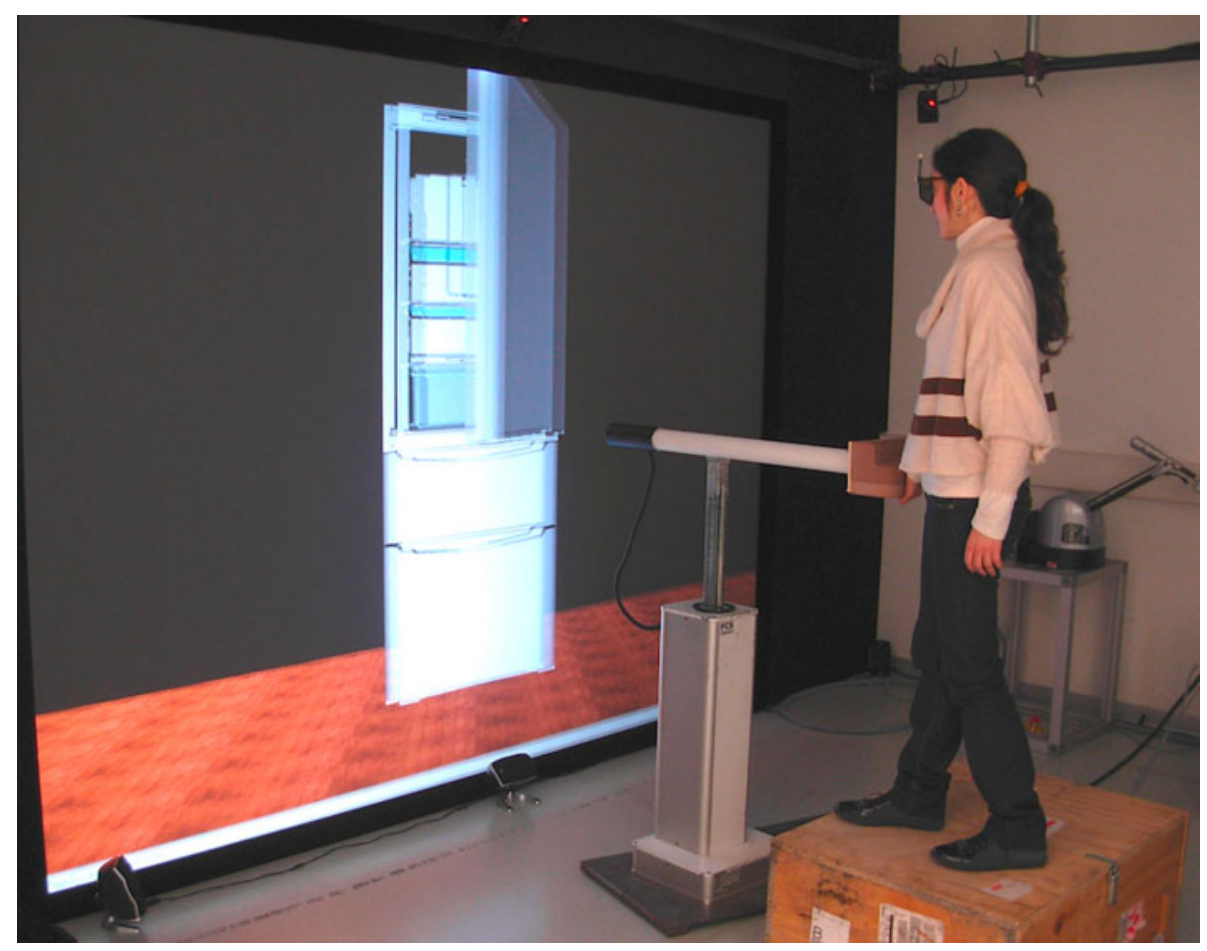

Figure 11. The iVP of a refrigerator door (Ferrise et al. 2013, Graziosi et al. 2013).

(2013) and the same approach used for the virtual simulation of a dishwasher door is reported in Graziosi et al. (2014).

The full physical description of the refrigerator door is fundamental in order to transform the information gathered from the PMU into detailed design specifications. In particular, Graziosi et al. (2014) describes thoroughly the process of mapping the results obtained from the tests with the interactive virtual prototype back to the physical description in the LMSAMESim environment.

The emotion evaluation is based on a psychological method, where users are asked to self-report their feeling about the experience (Bradley and Lang 1994). Future directions are toward the integration of physiological methods into the interactive virtual prototyping, detecting emotions by processing human body measured parameters, such as heart rate and skin conductance. These methods have been discarded at the moment, since they are typically used on static postures of the user, while in our experiments the users interact dynamically with the interactive virtual prototype.

\subsection{Experimental procedure}

In order to run a preliminary experiment to test the methodology, we have realised four configurations of the interactive virtual prototype, implementing different behaviours of a door when closing, as reported in Table 1.

An expert designer, with decades of experience in product design, has been asked to test the four door effects, one after the other. The designer started testing an initial configuration, and he was asked to change the parameters in order to reach a satisfactory behaviour for that effect. When satisfactory, the designer was asked to fill in a self-assessment manikin. He was asked to state the degree of satisfaction and the reason that led to request changes from the basic configuration.

Table 1. Four different tunable configurations of the interactive virtual prototype.

\begin{tabular}{|c|c|c|}
\hline Effect & Short description & Parameters that can be modified \\
\hline No force required & $\begin{array}{l}\text { The door seems weightless and closes without the } \\
\text { necessity of imparting a force }\end{array}$ & Starting from a null force response, it is possible to add and increase a viscosity effect \\
\hline Click effect & $\begin{array}{l}\text { When the door closes a click effect is perceived by } \\
\text { the user }\end{array}$ & It is possible to change the parameters setting the force feedback of the click \\
\hline Soft closing & The door closes with a soft effect & In this case the soft closing corresponds to a damping effect, whose value can be increased \\
\hline Automatic closing & $\begin{array}{l}\text { The door closes automatically after the door has reached a } \\
\text { defined angle }\end{array}$ & $\begin{array}{l}\text { This configuration corresponds to a torsional spring acting after a certain angle. The spring } \\
\text { stiffness can be increased or decreased }\end{array}$ \\
\hline
\end{tabular}


At the end of the tests, we were able to set a correlation between the physical characteristic of the virtual prototype of the refrigerator door, including the values of the parameters defining its behaviour, and the kind of satisfaction and emotional reactions elicited in the designer. The correlation of the physical characteristics of the product with the emotional response is information that is valuable for the design of optimised products, from a perceptual point of view.

Although not thoroughly validated, this methodology can be used to gather information about the correlation between physical characteristics of products and emotional responses, involving groups of users large enough for understanding which can be a pleasant behaviour shared by most of the users involved.

\section{Conclusion}

The paper has presented an emotional engineering methodology using interactive virtual prototyping for evaluating the user experience and the emotional response with newly designed products early in the development process.

The research is motivated by the fact that in the product development process one of the crucial phases is the evaluation of the design of the product that must satisfy the marketing research and the users' needs analysis. Marketers consider it important that users like a product during its daily use, for that creates affection to the brand and to the specific product, but also at the purchasing moment, for this affects the decision of buying that product instead of others.

Therefore, in the phase of ideation of a new product, it is crucial to test functionality and performances, as well as the interaction and experience of the users with the products, especially when dealing with the design of consumer products. The paper has presented some recent studies about the user's emotional reaction when interacting and experiencing products. Emotional feelings towards a product are correlated with the appreciation of the product and of its attributes. In so far as we are able to understand the human emotional response with respect to some product attributes, one can plan to tune those attributes until the emotional response is the desired (positive) one. In order to be sustainable, the users' evaluation and the emotional studies must be performed early in the development phase.

The paper has discussed the limitations of using physical prototyping for the evaluation of the design of new products. Because of these limitations, the authors propose a validation methodology based on interactive virtual prototyping, where the model of the product being evaluated and its behaviour are based on parametric descriptions driving a perceptual mock up. The parameter values, in terms of visual, haptic and sound effects, can be changed for setting and varying the behaviour experienced by the user during the interaction, which is supported by a multi-physics simulation tool.
The methodology has been evaluated through a preliminary test performed with an experienced designer. The methodology allows us to gather information about the correlation between physical characteristics of products and emotional responses. This capability has been considered important and beneficial when designing new products. In fact, in perspective, this methodology allows us to experiment and validate a new product with future consumers at the very early stage of the design process. This approach allows us to capture the best final behaviour perceived, and to characterise it in terms of physical, quantitative parameters. Those are passed to the design team as technical specifications for the detailed design. In this way, the risk of designing good functional products which are unsatisfactory from the point of view of the experience of use by the potential consumers is minimised, and in addition development time and costs are reduced.

\section{References}

Barrett, L.F., et al., 1998. Are women the 'more emotional' sex? Evidence from emotional experiences in social context. Cognition and Emotion, 12 (4), $555-578$.

Beckert, J. and Aspers, P., eds., 2011. The worth of goods: valuation and pricing in the economy. Oxford University Press.

Bezat, M.C., et al., 2007. Car door closure sounds: characterization of perceptual properties through analysis-synthesis approach. In: Proceedings of the 19th international congress on acoustics, 2-7 September 2007, Madrid, Spain.

Blessing, L.T. and Chakrabarti, A., 2009. DRM: a Design Research Methodology. London: Springer.

Bordegoni, M., 2011. Product virtualization: an effective method for the evaluation of concept design of new products. In: M. Bordegoni and C. Rizzi, eds. Innovation in product design. London: Springer, 117-141.

Bordegoni, M., Cugini, U., and Ferrise, F., 2013. Multisensory user experience design of consumer products. In: S. Fukuda, ed. Emotional engineering vol. 2. London: Springer, 219-242.

Bradley, M.M. and Lang, P.J., 1994. Measuring emotion: the self-assessment manikin and the semantic differential. Journal of Behavior Therapy and Experimental Psychiatry, 25 (1), 49-59.

Bradley, M.M. and Lang, P.J., 2006. Emotion and motivation. In: J. Cacioppo, L. Tassinary, and G. Berntson, eds. Handbook of psychophysiology. 2nd ed. Cambridge University Press, 581-607.

Cacioppo, J.T., et al., 2000. The psychophysiology of emotion. In: M. Lewis and H.J.J.M., eds. Handbook of emotions. New York: Guildford Press, 173-191.

Castellano, G., et al., 2008. Automated analysis of body movement in emotionally expressive piano performances. Music Perception: an Interdisciplinary Journal, 26 (2), 103-119.

Cuffaro, D. and Zaksenberg, I., 2013. The industrial design reference and specification book: everything industrial designers need to know every day. Beverly, MA: Rockport Publishers.

Damasio, A., 1994. Descartes' error: emotion, reason, and the human brain. New York: Avon Books.

Demir, E., Desmet, P.M.A., and Hekkert, P., 2009. Appraisal patterns of emotions in human-product interaction. International Journal of Design, 3 (2), 41-51.

Ekman, P., 1999. Basic emotions. In: T. Dalgleish and M. Power, eds. Handbook of cognition and emotion. Chichester: John Wiley \& Sons, 45-60. 
Ekman, P., 2007. Emotions revealed, second edition: recognizing faces and feelings to improve communication and emotional life. New York, NY: 2nd Holt.

Ekman, P. and Friesen, W.V., 1978. Facial action coding system: a technique for the measurement of facial movement. Palo Alto, CA: Consulting Psychologists Press.

Ekman, P., Levenson, R.W., and Friesen, W.V., 1983. Autonomic nervous system activity distinguishes among emotions. Science, 221 (4616), 1208-1210.

Ferrise, F., Bordegoni, M., and Graziosi, S., 2013. A method for designing users' experience with industrial products based on a multimodal environment and mixed prototypes. Computer-Aided Design and Applications, 10 (3), 461-474.

Field, T., 2003. Touch. Cambridge, MA: The MIT press.

Field, T.M., 1995. Touch in early development. New York, NY: Lawrence Erlbaum Associates.

Fukuda, S., ed., 2011. Emotional engineering: service development. London: Springer-Verlag.

Fukuda, S., ed., 2013. Emotional engineering vol. 2. London: Springer-Verlag.

Gallace, A. and Spence, C., 2013. In touch with the future. Oxford University Press.

Gatti, E., et al., 2013. Can the feel of the haptic interaction modify a user's emotional state? In: World haptics conference (WHC), 2013, 14-17 April 2013, Daejeon, Korea, 247-252.

Grandjean, D. and Scherer, K.R., 2008. Unpacking the cognitive architecture of emotion processes. Emotion, 8 (3), 341-351.

Graziosi, S., et al., 2013. A method for capturing and translating qualitative user experience into design specifications: the haptic feedback of appliance interfaces. In: DS 75-7: proceedings of the 19th international conference on engineering design (ICED13), Design for harmonies, Vol.7: human behaviour in design, 19-22 August 2103 Seoul, Korea, 427-436.

Graziosi, S., et al., 2014. Reverse engineering of interactive mechanical interfaces for product experience design. Virtual and Physical Prototyping, 9 (2), 65-79.

Hassenzahl, M., 2013. User experience and experience design. In: M. Soegaard and R.F. Dam, eds. The Encyclopedia of human-computer interaction. 2nd ed. Aarhus: The Interaction Design Foundation.

Hassenzahl, M. and Tractinsky, N., 2006. User experience - A research agenda. Behaviour and Information Technology, 25 (2), 91-97.

Johnstone, T., et al., 2005. Affective speech elicited with a computer game. Emotion, 5 (4), 513-518.

Juslin, P.N. and Scherer, K.R., 2005. Vocal expression of affect. In: J.A. Harrigan, R. Rosenthal, and K.R. Scherer, eds. The new handbook of methods in nonverbal behavior research. Oxford University Press, 65-135.
Kahneman, D. and Tversky, A., 2000. Choices, values, and frames. Cambridge University Press.

Lu, W. and Petiot, J.F., 2012. A sound-based protocol to study product emotions. International Journal of Product Development, 16 (3), 207-224.

Marr, D., 1982. Vision: a computational investigation into the human representation and processing of visual information. New York, NY: Henry Holt.

Mauss, I.B. and Robinson, M.D., 2009. Measures of emotion: a review. Cognition and Emotion, 23 (2), 209-237.

Nagamachi, M., 1995. Kansei engineering: a new ergonomic consumer-oriented technology for product development. International Journal of Industrial Ergonomics, 15 (1), 3-11.

Nagamachi, M., 1997. Kansei engineering: the framework and methods. In: M. Nagamachi, ed. Kansei Engineering 1. Tokyo: Kaibundo Publishing, 1-9.

Norman, D., 2004. Emotional design: why we love (or hate) everyday things. New York, NY: Basic Civitas Books.

Osgood, C.E., Suci, G., and Tannenbaum, P., 1957. The measurement of meaning. University of Illinois Press.

Petiot, J.F., Kristensen, B.G., and Maier, A., 2013. How should an electric vehicle sound? User and expert perception. In: Proceedings of the 2013 ASME international design engineering technical conferences and computers and information in engineering conference, August 4-7, Portland, OR.

Rao, A.R. and Monroe, K.B., 1989. The effect of price, brand name, and store name on buyers' perceptions of product quality: an integrative review. Journal of Marketing Research, 26 (3), 351-357.

Roozenburg, N. and Eekels, J., 1995. Product design: fundamentals and methods. Vol. 2. Chichester: John Wiley \& Sons.

Schacter, D., Gilbert, D., and Wegner, D., 2011. Sensation and perception, Psychology edition. New York: Worth Publishers, 166-171.

Scherer, K.R., 1984. On the nature and function of emotion: a component process approach. In: K.R. Scherer and P. Ekman, eds. Approaches to emotion. Hillsdale, NJ: L. Erlbaum Associates, 293-317.

Scherer, K.R., 2001. Appraisal considered as a process of multilevel sequential checking. In: K.R. Scherer, A. Schorr, and T. Johnstone, eds. Appraisal processes in emotion: theory, methods, research. New York and Oxford: Oxford University Press, 92-120.

Stompff, G., 2003. The forgotten bond: brand identity and product design. Design Management Journal (Former Series), 14 (1), 26-32.

Zurloni, V., et al., 2011. Addressing emotions within e-learning systems. In: I. Association, ed. Instructional design: concepts, methodologies, tools and applications. Hershey, PA: Information Science Reference, 1245-1258. 